\title{
Perfil do consumidor de aplicativos de comida delivery no município de Marabá-PA
}

\author{
Consumer profile of food delivery apps in the city of Marabá-PA \\ Perfil de consumidor de aplicaciones de comida a domicilio en la ciudad de Marabá-PA
}

Recebido: 30/12/2021 | Revisado: 04/01/2022 | Aceito: 14/01/2022 | Publicado: 16/01/2022

\author{
Maria Beatriz Martins Conde \\ ORCID: https://orcid.org/0000-0001-5718-0098 \\ Universidade do Estado do Pará, Brasil \\ E-mail: maria.conde@aluno.uepa.br \\ Gisele Rodrigues de Sousa \\ ORCID: https://orcid.org/0000-0002-0027-9414 \\ Universidade do Estado do Pará, Brasil \\ E-mail: gisele.sousa@aluno.uepa.br \\ Inês Clarissa Gomes Sousa \\ ORCID: https://orcid.org/0000-0002-5968-5652 \\ Universidade do Estado do Pará, Brasil \\ E-mail: ines.sousa@aluno.uepa.br \\ Antonia Caroline Pinho Vieira \\ ORCID: https://orcid.org/0000-0002-8245-6874 \\ Universidade do Estado do Pará, Brasil \\ E-mail: antonia.vieira@aluno.uepa.br \\ Jaqueline Ferreira da Silva \\ ORCID: https://orcid.org/0000-0001-7569-624X \\ Universidade do Estado do Pará, Brasil \\ E-mail: Jaqueline.fsilva@ aluno.uepa.br \\ Vitória Nazaré Costa Seixas \\ ORCID: https://orcid.org/0000-0003-0716-3876 \\ Universidade do Estado do Pará, Brasil \\ E-mail: vitoria.seixas@uepa.br
}

\begin{abstract}
Resumo
Recentemente a utilização do serviço delivery aumentou, passando a ser algo constante na rotina da sociedade, principalmente durante a pandemia da covid-19, devido ao isolamento e a restrição de algumas atividades. Tendo em vista a importância do comportamento e perspectiva para plataformas digitais, este artigo estudou o comportamento de expectativa do consumidor em relação ao delivery do município de Marabá- PA. Trata-se de uma pesquisa descritiva no qual 122 pessoas entrevistadas responderam um questionário online com 16 perguntas fechadas. Os resultados foram analisados utilizando- se planilhas eletrônicas elaboradas no Excel da Microsoft 2010. Dentre os principais resultados obtidos, destacam-se a viabilidade positiva em relação ao consumidor escolher a qualidade dos alimentos como critério principal, e o interesse do mesmo a respeito da limpeza dos estabelecimentos, assim como o alto índice de pessoas que desconhecem as condições higiênico-sanitárias dos estabelecimentos, deste modo notam-se a importância dos aplicativos de verificar os restaurantes antes de incluir na plataforma, para que consiga transmitir confiabilidade ao consumidor.
\end{abstract}

Palavras-chave: Comportamento; Higienização; Aplicativo.

\begin{abstract}
Recently, the use of delivery services increased, becoming something constant in society, mainly during the covid-19 pandemic, due to isolation and the restriction of some activities. In view of the importance of behavior and perspective for digital platforms, this article studies the behavior of consumer expectation in relation to delivery from the municipality of Marabá-PA. This is a descriptive research not qualifying 122 people interviewed will answer an online questionnaire with 16 dated questions. The results were analyzed using electronic templates prepared in Excel from Microsoft 2010. Among the main results obtained, the positive viability in relation to the consumer choosing the quality of two foods as the main criterion stands out. Establishments, as well as a high index of people that disregard the hygienic-sanitary conditions of two establishments, in this way note the importance of two applications to verify the restaurants before including the platform, so that it can transmit reliability to the consumer.
\end{abstract}

Keywords: Behavior; Sanitation; Application.

\section{Resumen}

Recientemente, se ha incrementado el uso del servicio de delivery, convirtiéndose en algo constante en la rutina de la sociedad, especialmente durante la pandemia del covid-19, debido al aislamiento y restricción de algunas actividades. En vista de la importancia del comportamiento y la perspectiva para las plataformas digitales, este artículo estudió el 
comportamiento de la expectativa del consumidor en relación a la entrega en el municipio de Marabá-PA. Se trata de una investigación descriptiva en la que 122 personas entrevistadas respondieron un cuestionario online con 16 preguntas cerradas. Los resultados se analizaron mediante planillas elaboradas en Microsoft Excel 2010. Entre los principales resultados obtenidos, se destaca la viabilidad positiva en relación a que el consumidor elija la calidad de los alimentos como criterio principal, y el interés del mismo en cuanto a la limpieza de los establecimientos, así como el alto índice de personas que desconocen las condiciones higiénico-sanitarias de los establecimientos, de ahí la importancia de las aplicaciones de verificación de los restaurantes antes de incluirlos en la plataforma, para que esta transmita confiabilidad al consumidor.

Palabras clave: Comportamiento; Saneamiento; Aplicación.

\section{Introdução}

Nos últimos tempos, a mudança na rotina da população levou-se a prática do consumo de comida através do sistema delivery, devido a obtenção da alimentação sem sair de casa ou do ambiente onde esteja, é um ramo que vem crescendo muito e com isso é necessário que o serviço de alimentação ofereça qualidade no atendimento, agilidade, higiene e sabor da comida (Sebrae, 2017).

Devido a acessibilidade à internet, os aplicativos de comida delivery estão ganhando espaço no mercado alimentício, em virtude da praticidade e comodidade que a ferramenta oferece, no qual o consumidor escolhe a comida de sua preferência, no conforto do seu lar, sem precisar enfrentar filas, além de apreciar diferentes tipos de culinária, entre outras vertentes que o aplicativo disponibiliza, também o mesmo possibilita ao cliente que os seus históricos de pedidos anteriores fiquem salvos (Monty, 2018; Menighini et al., 2021).

Durante a pandemia da Covid-19 o uso do delivery intensificou, pois muitos estabelecimentos, inclusive restaurantes, foram fechados para o atendimento presencial com a finalidade de evitar a disseminação do vírus, e com isso o ambiente digital passou a ser a ferramenta utilizada para as vendas de alimentos (Santana et al., 2021).

Diante do exposto, o intuito do presente trabalho e avaliar o perfil do consumidor de aplicativos de comida delivery no município de Marabá-PA.

\section{Materiais e Métodos}

O presente estudo tratou-se de uma pesquisa descritiva, no qual a mesma tem como objetivo primordial a descrição das características de determinada população ou fenômeno ou o estabelecimento de relações entre variáveis, conforme explica Gil (1999).

A pesquisa foi desenvolvida no período de 23 de março a 12 de abril 2021 na cidade de Marabá-PA, realizou-se uma entrevista com 122 pessoas, um questionário online contendo 16 perguntas fechadas, conforme mostra o Quadro 1, utilizando a plataforma Google Forms, foi disponibilizado para estes usuários de aplicativo de comida delivery em rede social via WhatsApp. Para a realização da entrevista foram selecionadas pessoas que residem no município de Marabá-PA que tenham o hábito de utilizar este serviço.

Realizou-se uma análise descritiva apresentada em forma de frequência das respostas e a porcentagem referente a cada uma das categorias. Os dados coletados dos questionários foram registrados e tabulados em planilhas eletrônicas elaboradas no Excel da Microsoft 2010 
Quadro 1 - Questionário aplicado na avalição dos aplicativos delivery. Marabá, 2021 (n=122).

\begin{tabular}{|c|c|}
\hline $\mathbf{1}$ & Gênero \\
\hline $\mathbf{2}$ & Faixa etária \\
\hline $\mathbf{3}$ & Ocupação \\
\hline $\mathbf{4}$ & Com quem os consumidores residem \\
\hline $\mathbf{5}$ & Aplicativo de comida delivery mais utilizado \\
\hline $\mathbf{6}$ & Frequência utilizada \\
\hline $\mathbf{7}$ & Critério utilizado na hora de comprar \\
\hline $\mathbf{8}$ & Motivos que leva o consumido utilizar o aplicativo de comida muitas vezes durante a semana \\
\hline $\mathbf{9}$ & Se o consumidor conhece as condições higiênico-sanitárias do estabelecimento alimentício \\
\hline $\mathbf{1 0}$ & O interesse do consumidor em saber as condições de limpeza do estabelecimento \\
\hline $\mathbf{1 1}$ & Se o consumidor tem hábito de ser exigente com a qualidade higiênica da comida \\
\hline $\mathbf{1 2}$ & Quando a comida chega na sua residência se ele observa se está lacrada \\
\hline $\mathbf{1 3}$ & Em relação à temperatura da comida que foi pedida como chegar na casa do consumidor \\
\hline $\mathbf{1 4}$ & Tempo que demora para comida chegar \\
\hline $\mathbf{1 5}$ & Tipo de comida mais pedida \\
\hline $\mathbf{1 6}$ & O que o consumidor faz quando acontece algum problema com a comida \\
\hline
\end{tabular}

Fonte: Autores.

\section{Resultados e Discussões}

Os resultados deste estudo em relação ao gênero do consumidor, sendo (60\%) mulheres e (40\%) homens, semelhante ao encontrado por França (2021), (60\%) dos usuários do delivery são mulheres, sejam em capitais, assim como no recorte nacional e no interior de acordo com sua pesquisa sobre os números do delivery online no Brasil em 2020.

A faixa etária do consumidor neste estudo corresponde a (51\%) são jovens de até 21 anos, (25\%) pessoas de 22 a 30 anos, (17\%) de 31 a 45 anos, (5\%) de 46 a 55 anos, a minoria sendo (2\%) dos consumidores. Ao contrário da pesquisa realizada por Tandon et al. (2021), no qual (28,7\%) dos consumidores delivery, são adultos com idade entre 26 a 30 anos.

A ocupação do público entrevistado no município de Marabá, (27\%) trabalham e estudam, (25\%) estudam em período parcial, (13\%) estudam em período integral, (17\%) trabalham em período parcial, (13\%) em período integral e (4\%) têm outras ocupações, diferente da pesquisa realizada por McCarthy (2020), os principais clientes de entrega de comida geralmente são funcionários em tempo integral ou profissionais que trabalham em casa.

Questionados com quem os mesmos residem (56\%) com os pais e irmãos, (19\%) cônjuge e filhos, (10\%) moram sozinhos, (9\%) com um companheiro (a), (6\%) parentes e amigos, estes resultados diferem do estudo de Clientesa (2016), no qual (41\%) possui a companhia de cônjuge e filhos (41\%), os que moram com amigos ou outros parentes (29\%) e aqueles que moram somente com algum companheiro (18\%), somente (8\%) dos consumidores moram sozinhos, ficando na quarta posição.

Em relação ao aplicativo de comida mais utilizado neste estudo, (42\%) deste público preferem as redes sociais para realizar seus pedidos, (41\%) Ifood, (10\%) utilizam outros aplicativos, (5\%) Tô no lucro, sendo (2\%) 99 store, resultados expressos nestes estudos são convergentes com a pesquisa de Verissimo et al. (2017), nos quais os autores demostram a totalização de 125 respondentes $(91,9 \%)$ contra 11 respondentes $(8,1 \%)$ que nunca usou algum tipo de aplicativo móvel.

As pessoas interrogadas neste estudo dentre as quais estão (47\%) possuem o hábito de pedir comida aos finais de semana, assim cerca de (17\%) uma vez na semana, bem como (17\%), duas vezes na semana, (7\%) três vezes na semana, $(7 \%)$ durante a semana (5\%) em outra frequência, resultados similares foi encontrado na pesquisa do Instituto QualiBest (2020), da qual os pesquisadores destacaram que a maior parte dos usuários costumam pedir delivery de comida aos finais de semana, sendo corresponde ao total de $(67,3 \%)$. 
Quanto os critérios utilizados pelas pessoas entrevistadas na hora de efetuar a compra (36\%) responderam que a qualidade dos alimentos é uma questão que sobressai, (20\%) a vontade de comer o alimento, (18\%) cupom/ promoção, (14\%) preço, (10\%) taxa de entrega, (2\%) possui outros motivos, neste estudo os resultados encontrados verificaram que o fator qualidade é mais importante do que os estudos evidenciados por Israel e Velu (2019), em que indicaram (42,7\%) dos consumidores escolhem o delivery de comida devido a entrega fácil.

Em relação aos consumidores entrevistados nesta pesquisa relataram os motivos que levam eles a utilizarem o delivery muitas vezes durante a semana, (42\%) não precisar sair de casa, $(24 \%)$ possibilidade de comer qualquer tipo de comida, (17\%) praticidade por não ter que cozinhar, (6\%) falta de tempo para pensar em algo para cozinhar, (6\%) morar sozinho (a), os resultados desta pesquisa opõem o estudo realizado por Silva (2019), que ao analisar sobre o que influencia a escolher tais aplicativos o motivo primordial foi a facilidade no manuseio do total de (56\%), assim pode-se evidenciar que nas outras respostas temos resultados dispares quando comparado a esse atual estudo (18\%) alegaram a diversidade de estabelecimentos, (7\%) escolhem o aplicativo pela qualidade, (5\%) preferem pela confiabilidade, (6\%) o que os influenciou foi a segurança, (4\%) preferem pela rapidez e (4\%) preferem por outros motivos.

Conforme os conhecimentos dos consumidores em relação às condições higiênico-sanitárias, (46\%) relataram que não conhecem as condições higiênico-sanitárias dos estabelecimentos, (31\%) afirmaram conhecer e (23\%) talvez, os resultados evidenciados neste trabalho se assemelham com a pesquisa de Fonseca et al. (2018), realizada em São Luís - MA a respeito do conhecimento dos consumidores de comida de rua sobre segurança dos alimentos, no qual os consumidores destes pontos de vendas demostraram ter conhecimentos regulares sobre segurança dos alimentos.

Em relação ao saber sobre a limpeza dos estabelecimentos, $(85 \%)$ responderam ter interesse em saber sobre as boas condições do ambiente e (15\%) não tem interesse, a preocupação dos consumidores tende a ser cada vez mais evidente em relação à limpeza dos estabelecimentos resultados equivalentes foram encontrados no estudo feito pela Da Redação (2020), baseado em uma pesquisa do Instituto QualyBest, no qual relatam que a pandemia da covid-19 mudou o comportamento do consumidor, neste contexto, os critérios para a escolha de restaurantes mudaram, sendo a importância de o estabelecimento oferecer uma comida gostosa aparece em terceiro lugar, sendo indicada por (43\%), dos respondentes. Assim, a preocupação com limpeza e higiene é campeã, citada por (78\%).

Os consumidores, (84\%) revelaram que são exigentes com a questão da qualidade higiênica da comida que compra, (9\%) responderam que talvez, enquanto (7\%) disseram não, resultados medianos foram encontrados na pesquisa de Pinheiro et al. (2011), sobre perfil de consumidores em relação à qualidade de alimentos e hábitos de compras a respeito da qualidade, o item higiene foi descrito como uns dos aspectos ligados à determinação de qualidade dos alimentos pelos entrevistados deste estudo, sendo (33,1\%), predominando o item nutrição com (46,9\%).

Em relação a observar se a embalagem está lacrada, (86\%) das pessoas interrogadas verificam se estão em conformidade, os outros (7\%) alegaram talvez e outros (7\%) disseram que não observam. Os resultados indicados neste trabalho refletem a pesquisa de Viana \& Muniz (2020), no seu estudo avaliou-se que os empresários do ramo alimentício devem garantir ao realizar a entrega, acomodação em embalagens adequadas, íntegras e limpas, inclusão de informações sobre o consumo do alimento (ex. rotulagem informando para consumir imediatamente ou orientação de aquecimento) e lacres de segurança.

Este estudo (52\%) dos entrevistados, disseram que ao pedir, o alimento chega morno, (45\%) alegaram que chega quente e (3\%) fria, respostas equivalentes foram identificadas no trabalho desenvolvido por Beltrão (2019), em sua pesquisa sobre análise dos serviços de delivery de hambúrgueres artesanais, (90\%) dos hambúrgueres foram entregues em uma temperatura ótima para o consumo. 
Os resultados expressos sobre o tempo que demora a comida chegar à residência do consumidor (54\%) disseram que o pedido demora em torno de 30 min há 1 hora para chegar, (22\%) em média de 30 minutos, (12\%) de 1 a 2 horas e os outros (12\%) menos de 30 minutos, os resultados encontrados se assemelham com a pesquisa realizada pelo site Anota ai (2019), na qual o cliente não apenas quer o melhor produto, como também exige uma entrega rápida e eficiente.

Dos entrevistados (45\%) tem o hábito de pedir pizza, (38\%) hambúrgueres, (12\%) churrasco, (2\%) sobremesas, (3\%) outros tipos de comidas. Os resultados comprovados nesta pesquisa contrariam a pesquisa de França (2021), entre as quais os lanches como sanduíches, calzones, esfihas e batatas fritas foram às refeições mais pedidas em 2020 pelo serviço delivery, devido à praticidade a agilidade.

Os consumidores relatam qual atitude costuma tomar quando ocorre algum problema com a comida (55\%) responderam que reclamam com os proprietários dos estabelecimentos, (29\%) reclamam no aplicativo de comida, (10\%) pedi o dinheiro de volta, (3\%) expõe em redes sociais, $(2 \%)$ toma outras medidas, (1\%) fazem denuncia na vigilância sanitária, os resultados descobertos neste estudo, estão de acordo com o Mundo dos Advogados (2018), os clientes podem exigir seus direitos quando o serviço não é prestado de forma satisfatória, sendo assim o Código de Defesa do Consumidor ampara os clientes nesse sentido, impondo aos estabelecimentos providências quando não cumprem com a qualidade do produto, exatidão do pedido e prazo de entrega.

\section{Conclusão}

Diante das informações coletadas neste estudo, observou-se que possui pouca disponibilidade de informações para os consumidores com relação ao serviço delivery, principalmente em relação as condições higiênico-sanitárias dos estabelecimentos, deste modo é necessário que os aplicativos sejam mais minuciosos com a questão higiênica dos mesmos juntamente com os órgãos fiscalizadores, verificando a situação antes de introduzir na plataforma digital.

Para estudos futuros espera-se realizar pesquisa sobre as condições higiênico-sanitárias de estabelecimentos que utilizam os aplicativos de comida como comercialização, avaliando as boas práticas de manipulação.

\section{Referências}

Anota aí. (2019). Como reduzir o tempo de entrega do delivery. https://anota.ai/blog/como-reduzir-o-tempo-de-entrega-do-delivery/

Beltrão, L. B. D. A. (2019). Análise dos serviços de delivery de hambúrgueres artesanais via aplicativo na Universidade Federal Rural de Pernambuco. Trabalho de conclusão de curso (Gastronomia) da Universidade Federal Rural de Pernambuco. http://hdl.handle.net/123456789/2113

Clientesa. (2016). Quem é o comprador de delivery? https://www.clientesa.com.br/estatisticas/62354/quem-e-o-comprador de-delivery

Controlare, (2021). Cozinhas de delivery têm regularização própria? https://www.controlare.com.br/blog/seguranca-alimentar/cozinhas-de-delivery-temregularizacao-propria/

Mundo dos Advogados. (2018). Delivery: conheça os direitos do consumidor. https://www.mundoadvogados.com.br/artigos/delivery-conheca-os-direitos-doconsumidor

Da redação. (2020). Higiene supera sabor na hora de escolher restaurante. https://exame.com/marketing/higiene-supera-sabor-na-hora-de-escolher-restaurante/

Fonseca, W. C., Moreno, B. F. S., Silva, I. M. R., \& Lacerda, L. D. M. (2018). Comida de rua: conhecimento dos consumidores sobre segurança dos alimentos e condições higienicossanitárias de pontos de venda em São Luís, MA. Higiene. Alimentar, 32 (284/285), 61-65. https://pesquisa.bvsalud.org/portal/resource/pt/biblio-965434

França, I. (2021). 14 comidas mais pedidas em 2020 no Brasil. https://blog.deliverymuch.com.br/comidas-mais-pedidas-em-2020/

França, I. (2021). Pesquisa: números do delivery online no Brasil em 2020. https://blog.deliverymuch.com.br/numeros-do-delivery-online-no-brasil/

Gil, A. C. (2008). Métodos e técnicas de pesquisa social. 6. ed. Editora Atlas SA. https://ayanrafael.files.wordpress.com/2011/08/gil-a-c-mc3a9todos-etc3a9cnicas-de-pesquisa-social.pdf

Instituto Qualibest. (2020). Conheça o perfil dos usuários de aplicativos de delivery de comida. https://www.institutoqualibest.com/marketing/conheca-operfil-dos-usuarios-de-aplicativos-de-delivery-de-comida/ 
Israel, D. J., \& Velu, R. (2019). Consumer's intention to continuous use of mobile food delivery aggregator app'. Journal of Advanced Research in Dynamical and Control Systems, 11 (7), 119-128

Mccarthy, A. (2020). Rise in Food Delivery: Consumer Trends on Ordering. https://get.doordash.com/blog/rise-in-food-delivery-and-why-it-is-popular

Menighini, G. V., de Oliveira, J. C. C., de Cillos Silva, V., \& Piacente, F. J. (2021). Impacto da pandemia na demanda por aplicativo de delivery de alimentação em Piracicaba/SP. Research, Society and Development, 10(6), 28310615945-e28310615945. 10.33448 / rsd-v10i6.15945

Monty, R. (2018). Consumo de comida por aplicativos: os impactos das materialidades da comunicação em Uber Eats e iFood. Anais do Congresso Internacional Comunicação e Consumo (Comunicon), $7^{o}$ Encontro de GTs de Pós-Graduação. Rio de Janeiro, Brasil. http://anaiscomunicon.espm.br/GTs/GTPOS/GT2/GT02_MONTY.pdf

Pinheiro, F. de A., Cardoso, W. S., Chaves, K. F., de Oliveira, A. S. B., \& de Almeida Rios, S. (2011). Perfil de consumidores em relação à qualidade de alimentos e hábitos de compras. Journal of Health Sciences, 13(2), 95-102. https://doi.org/10.17921/2447-8938.2011v13n2p\%25p

Santana, A. G., da Costa, M. L. G., \& Shinohara, N. K. S. (2021). Alimentação em tempos de pandemia do Coronavírus: a ressignificação de uma prática cotidiana e dietética. Research, Society and Development, 10 (3), 50410313442-e50410313442. 10.33448/rsd-v10i3.13442

Sebrae. (2017). Delivery para restaurantes. https://www.sebrae.com.br/sites/PortalSebrae/artigos/delivery-para-restaurantes,20a97b008b1034 10VgnVCM10 0000b272010aRCRD

Silva, L. N., Pereira, G. T. J., Pales, H. C., de Fátima Santos, M., \& Silva, T. O. S. (2019). Qualidade percebida nos serviços ofertados por aplicativos de alimentos e bebidas em vitória da Conquista-Ba. Diálogos Interdisciplinares, 8(9), 1-18. https://revistas.brazcubas.br/index.php/dialogos/article/view/711

Soares, A. C. N., \& Lima, M. R. S. (2020). Food delivery services and their precautions in times of the SARS-VOC-2 Pandemic (COVID-19). Brazilian Journal of Health Review, 3 (3), 4217-4226. doi:10.34119/bjhrv3n3-025

Tandon, A., Kaur, P., Bhatt, Y., Mäntymäki, M., \& Dhir, A. (2021). Why do people purchase from food delivery apps? A consumer value perspective. Journal of Retailing and Consumer Services, 63, 102667. doi.org/10.1016/j.jretconser.2021.102667

Verissimo, P., Moretti, S. D. A., \& Oliveira, S. D. (2017). O uso de aplicativos móveis no setor de alimentos e bebidas: uma análise do comportamento do usuário de novas tecnologias. Perspectivas em Ciências Tecnológicas, 6 (6), 29-42

Viana, M. \& Muniz, Karyna (2020). Segurança dos Alimentos: Dicas de cuidado para o Delivery em meio a Covid-19. https://bibliotecas.sebrae.com.br/chronus/ARQUIVOS_CHRONUS/bds/bds.nsf/ed6f4e148e620b04690ebb81c133c7d9/\$File/19424.pdf 